Ann. Sci. forest., 1975, 32 (1), 1-16.

\title{
ÉTUDE DE LA CROISSANCE EN HAUTEUR CHEZ QUELQUES RÉSINEUX, EFFET DE LA TEMPÉRATURE
}

\author{
G. AUSSENAC \\ Station de Sylviculture et Production, \\ Centre national de Recherches forestières, I.N.R.A., \\ Champenoux - 54280 Seichamps

\section{RÉSUMÉ}

L'auteur a étudié la croissance en hauteur de quatre espèces de résineux : Abies alba, Abies nordmanniana, Picea abies, Pseudotsuga menziesii, poussant dans des conditions microclimatiques différentes.

A partir de mesures bihebdomadaires, il a étudié la relation qui existait entre la température moyenne de l'air et le coefficient de croissance :

$$
k=\frac{1}{l\left(1-\frac{l}{\mathrm{~L}}\right)} \cdot \frac{\Delta l}{\Delta t}
$$

où $l$ représente la hauteur de la pousse à l'instant $t$ et $\mathrm{L}$ la hauteur finale de la pousse.

Ce coefficient de croissance s'annule pour les températures suivantes :

$\begin{array}{ll}\text { Abies alba } & 5,2^{\circ} \mathrm{C} \\ \text { Abies nordmanniana } & 6,2^{\circ} \mathrm{C} \\ \text { Picea abies } & 4,1{ }^{\circ} \mathrm{C} \\ \text { Pseudotsuga menziesii } & 5,8^{\circ} \mathrm{C}\end{array}$

qui constituent les seuils apparents de végétation. Abies nordmanniana qui est l'espèce la plus « méridionale » a le seuil de végétation le plus élevé alors que Picea abies qui est l'espèce la plus septentrionale a le seuil de végétation le plus bas.

La croissance en hauteur a ensuite été étudiée en fonction des sommes de température calculées à partir des seuils apparents de végétation établis pour chacune des espèces.

Les courbes obtenues sont des courbes classiques en « $\mathbf{S}$ ». La pente de la tangente au point d'inflexion peut être considérée comme constante pour une même espèce. Les coefficients angulaires de ces droites sont les suivants :

$$
\begin{array}{ll}
\text { - Abies alba } & 0,332 \\
\text { - Abies nordmanniana } & 0,308 \\
\text { - Picea abies } & 0,336 \\
\text { - Pseudotsuga menziesii } & \mathbf{0 , 5 1 3}
\end{array}
$$

Ces résultats suggèrent que les potentialités de croissance de ces différentes espèces sont conditionnées par ces mécanismes d'action de la température. Le Douglas a la potentialité de croissance la plus importante, suivi par l'Épicéa et le Sapin pectiné; le Sapin de Nordmann arrivant en dernier. 


\section{1. - INTRODUCTION}

La croissance en hauteur des arbres forestiers est conditionnée par plusieurs facteurs écologiques. Parmi ceux-ci, les facteurs climatiques jouent un rôle particulièrement important et commandent les potentialités de croissance.

La croissance est la conséquence de l'activité photosynthétique, elle-même sous la dépendance de facteurs tels que le rayonnement solaire, l'alimentation en eau, la température. Elle est, en fait, toutes choses égales par ailleurs, l'expression des potentialités génétiques de l'espèce face aux conditions climatiques ou microclimatiques qui lui sont faites. Parmi les facteurs climatiques, la température a une action primordiale sur les phénomènes de développement des arbres. Les lois d'action de la température sur le développement des végétaux ont été étudiées et précisées par plusieurs auteurs.

Bien sûr, d'autres facteurs climatiques interviennent et d'une année à l'autre, la croissance en hauteur est variable et dépend à la fois des conditions de l'année précédente et de celle de l'année en cours (AussenAC, 1973). Dans la nature, il est alors difficile de dégager les lois générales de croissance des espèces forestières. Cependant, il est possible à l'aide de dispositifs expérimentaux adaptés et de mesures suffisamment fines de dégager quelques conclusions partielles.

\section{2. - DISPOSITIF EXPÉRIMENTAL}

L'étude entreprise porte sur 4 espèces résineuses dont les caractéristiques principales sont données dans le tableau suivant :

TABLEAU 1

Caractéristiques des espèces étudiées

\begin{tabular}{|c|c|c|c|}
\hline Espèces & Provenance & $\begin{array}{l}\text { Age }\left({ }^{1}\right) \\
\text { (ans) }\end{array}$ & $\begin{array}{l}\text { Nombre de plants } \\
\text { à la plantation }\end{array}$ \\
\hline Abies alba Mill. & $\begin{array}{l}\text { Ecouves II, Lat. } 48^{\circ} 31^{\prime} \text {. } \\
\text { Long. } 0^{\circ} 04^{\prime} \mathrm{E} \text {, Orne (France). } \\
\text { Altitude } 300 \mathrm{~m} \text {. }\end{array}$ & 9 & $\begin{array}{l}74 \text { Coupe d'abri } \\
63 \text { Clairière } \\
59 \text { Coupe rase }\end{array}$ \\
\hline $\begin{array}{c}\text { Abies nordmanniana } \\
\text { Spach. }\end{array}$ & $\begin{array}{l}\text { Gerbéviller, Lat. } 48^{\circ} 30^{\prime} \text {. } \\
\text { Long. } 6^{\circ} 30^{\prime} \mathrm{E} \text {, Meurthe-et-Moselle } \\
\text { (France). } \\
\text { Altitude } 300 \mathrm{~m} \text {. }\end{array}$ & 9 & $\begin{array}{l}44 \text { Coupe d'abri } \\
44 \text { Clairière } \\
55 \text { Coupe rase }\end{array}$ \\
\hline Picea abies L. & $\begin{array}{l}\text { La Joux, Lat. } 46^{\circ} 51^{\prime} \text {. } \\
\text { Long. } 6^{\circ} 03^{\prime} \mathrm{E}, \text { Jura (France). } \\
\text { Altitude } 800 \mathrm{~m} \text {. }\end{array}$ & 9 & $\begin{array}{l}63 \text { Coupe d'abri } \\
58 \text { Clairière } \\
57 \text { Coupe rase }\end{array}$ \\
\hline $\begin{array}{c}\text { Pseudotsuga menziesii } \\
\text { Mirb. }\end{array}$ & $\begin{array}{l}\text { Farges II, Lat. } 45^{\circ} 32^{\prime} . \\
\text { Long. } 2^{\circ} 11^{\prime} \mathrm{E} \text {, Corrèze (France). } \\
\text { Altitude } 700 \mathrm{~m} .\end{array}$ & 9 & $\begin{array}{l}64 \text { Coupe d'abri } \\
60 \text { Clairière } \\
53 \text { Coupe rase }\end{array}$ \\
\hline
\end{tabular}

(1.) (en 1971). 
Les plants ont été plantés à 4 ans dans trois milieux différents :

- sous une coupe d'abri constituée par un taillis de charmes, hêtres, chênes, d'une densité de 1300 tiges/ha, et d'une hauteur de $13 \mathrm{~m}$.

- dans une clairière circulaire de $26 \mathrm{~m}$ de diamètre située au sein d'un peuplement forestier ayant des caractéristiques identiques au précédent,

- en plein découvert dans une coupe rase.

Le dispositif expérimental est situé en forêt domaniale d'Amance à $15 \mathrm{~km}$ à l'Est de Nancy. Les conditions topographiques et climatologiques générales sont identiques : replat à $250 \mathrm{~m}$ d'altitude sur sol brun lessivé à pseudogley, sur marnes liasiques.

Les principaux facteurs microclimatiques ont été étudiés de 1967 à 1972. La température de l'air a été relevée dans chacun des trois milieux choisis à $1,50 \mathrm{~m}$ de hauteur. La croissance en hauteur de chacun des arbres a été mesurée deux fois par semaine tout au long de la saison de végétation (avril-août). A partir de ces données, il nous a été possible de calculer la croissance moyenne dans chacun des milieux. Les mesures ont été effectuées à l'aide d'un mètre gradué en millimètre, à partir de la base des bourgeons.

\section{3. - RÉSULTATS}

\section{1. - Variations de la croissance en fonction du temps}

Le rythme et l'importance de la croissance en hauteur des arbres ont donc été étudiés dans les trois traitements en effectuant des mesures périodiques tout au long de la saison de végétation (1967-1972). A partir de ces mesures, il a été possible d'établir des courbes de croissance en fonction du temps. Ce sont des courbes en $" S$ » caractéristiques. Ces courbes nous permettent de mettre en évidence l'effet des traitements étudiés. Les figures 1 et 2 par exemple, montrent ce que nous avons obtenu pour l'année 1969. L'étude de l'ensemble de ces courbes sera faite dans une publication ultérieure. On remarquera simplement, pour l'instant, que pour les quatre espèces étudiées : Abies alba, Abies nordmanniana, Picea abies et Pseudotsuga menziesii, la croissance en hauteur est plus importante dans la clairière que dans les autres traitements.

Ces courbes sont intéressantes pour faire apparaître l'effet des traitements, mais elles sont difficilement comparables d'une année à une autre. Il faut alors essayer de l'exprimer en fonction d'autres facteurs que le temps : la température ou le rayonnement solaire par exemple. Dans l'étude entreprise ici, l'effet du rayonnement est difficile à mettre en évidence car il reste relativement stable pendant la période de croissance des espèces étudiées qui se situe d'une façon générale pendant les mois de mai, juin et juillet. Par contre, l'effet de la température a pu être envisagé car d'une année à l'autre, les conditions thermiques peuvent être très variables pour une même date.

\section{2. - Action de la température sur la croissance en hauteur}

\subsection{Détermination des seuils apparents de végétation.}

A partir des mesures bi-hebdomadaires, nous avons étudié la relation qui existait entre la température moyenne journalière de l'air et l'accroissement moyen journalier. Mais, cette relation n'est pas très bonne, les coefficients de corrélation que nous avons obtenus sont faibles et souvent non significatifs. En fait, ceci s'explique aisément, ainsi que le fait remar- 

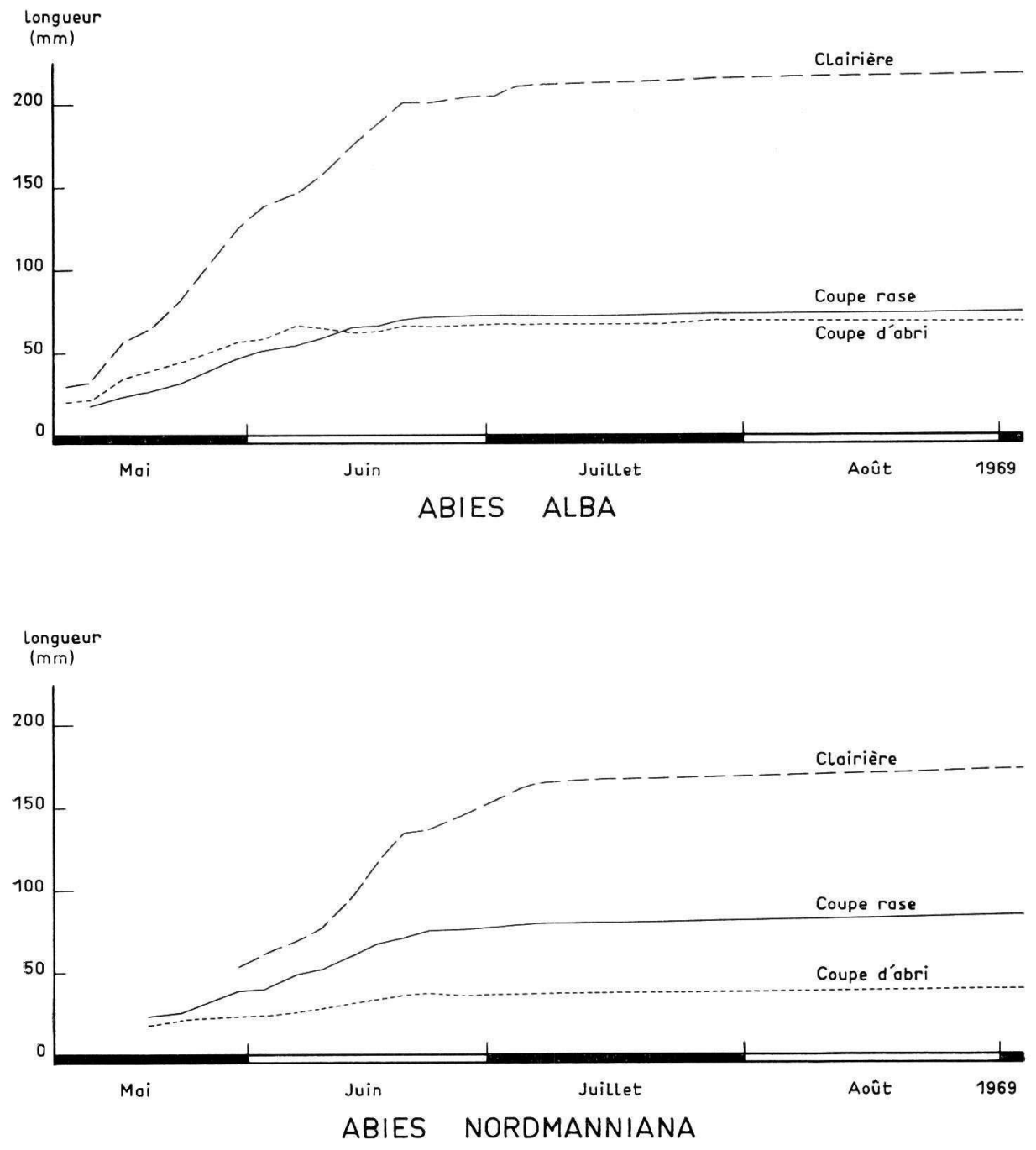

FIG. 1. - Variations de la longueur de la pousse en fonction du temps dans les différents traitements étudiés : clairière, coupe rase, coupe d'abri 

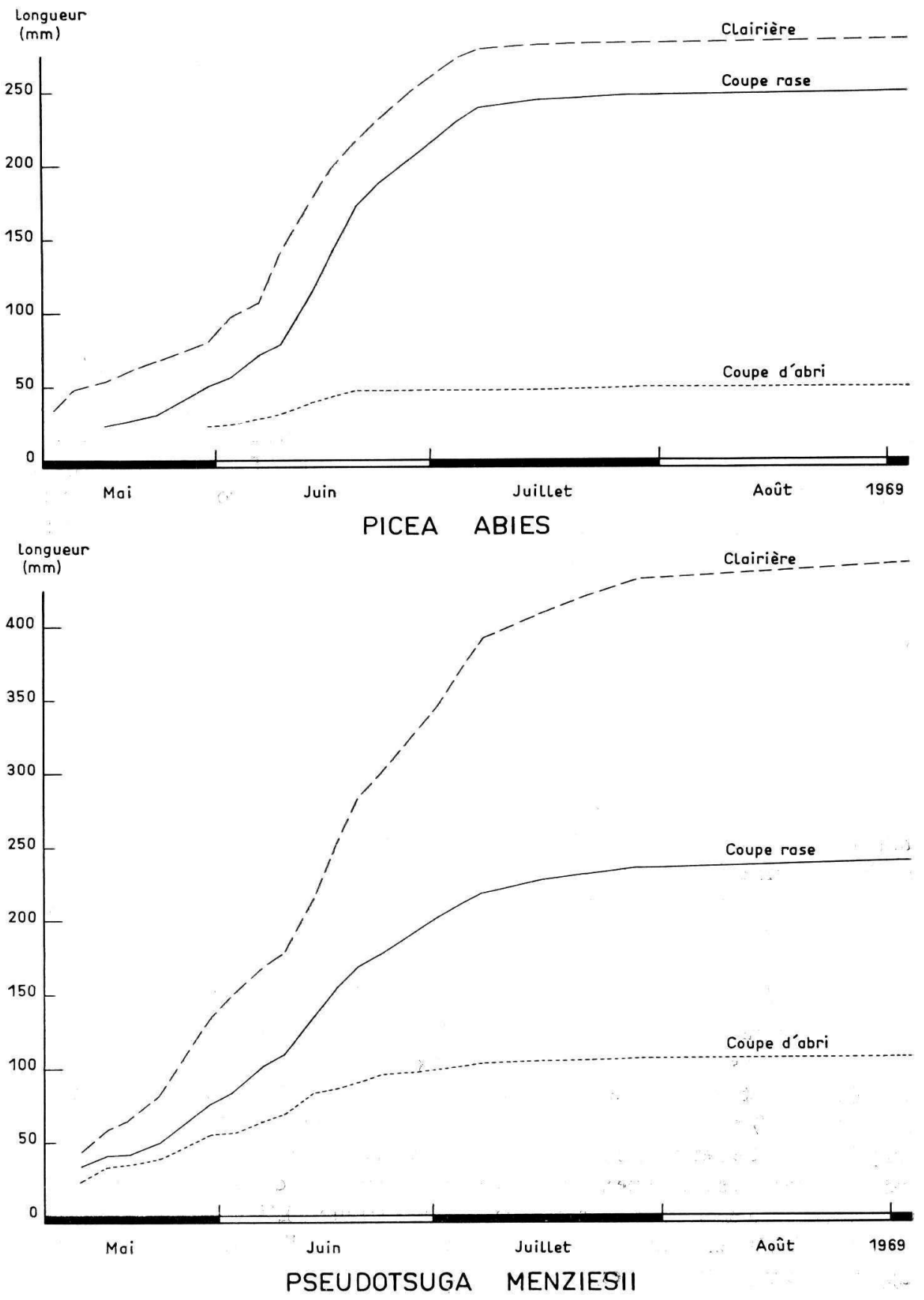

FIG. 2. - Variation de la longueur de la pousse en fonction du temps dans les différents traitements étudiés : clairière, coupe rase, coupe d'abri 
quer Durand et al (1967) si l'on tient compte du rythme interne d'évolution de la croissance. L'accroissement de la pousse dépend de sa taille. Robertson (1908) a proposé, pour exprimer l'accroissement, la relation suivante :

$$
\frac{\Delta 1}{\Delta \mathrm{t}}=k l\left(1-\frac{1}{\mathrm{~L}}\right)
$$

dans laquelle $l$ représente la taille de la pousse à l'instant $t$, L est la pousse totale, $k$ est un coefficient qui varie avec les facteurs écologiques ( $\left.{ }^{\mathbf{1}}\right)$. Il est dès lors possible, ainsi que l'ont fait Durand et al (1967), de calculer

$$
k=\frac{1}{l\left(1-\frac{l}{\mathrm{~L}}\right)} \cdot \frac{\Delta l}{\Delta t}
$$

Ce coefficient de croissance varie avec la température quand $k$ est nul $\frac{\Delta l}{\Delta t} \mathrm{~s}$ 'annule, l'accroissement est donc égal à zéro. La figure 3 donne pour les espèces étudiées les équations de régression qui traduisent la variation de $k$ en fonction des températures moyennes journalières ordonnées par valeurs croissantes. L'étude comparative de ces équations de régression à partir de la méthode proposée par DéCOURT (1971) nous a montré qu'elles ne sont pas toutes significativement différentes les unes des autres. Il est seulement apparu que d'une part Abies alba était différent de Abies nordmanniana et de Pseudotsuga menziesii et que d'autre part Picea abies était différent de Pseudotsuga menziesii. Le coefficient $k$ s'annule pour les températures suivantes :

$\begin{array}{ll}\text { - Abies alba } & 5,2^{\circ} \mathrm{C} \\ \text { - Abies nordmanniana } & 6,2^{\circ} \mathrm{C} \\ \text { - Picea abies } & 4,1^{\circ} \mathrm{C} \\ \text { - Pseudotsuga menziesii } & 5,8^{\circ} \mathrm{C}\end{array}$

Ces températures pour lesquelles $k$ est nul correspondent au seuil apparent de végétation (ou zéro de végétation). Des différences existent. Bien qu'elles ne soient pas toutes statistiquement différentes, il est intéressant de remarquer que Abies nordmanniana a un seuil apparent de végétation supérieur aux trois autres espèces. Par contre, l'Épicéa apparaît comme l'espèce la plus « froide ».

\subsection{Expression de la croissance en hauteur en fonction des sommes de températures.}

De nombreux auteurs ont essayé de lier la croissance à la température et plus précisément aux sommes de températures. Deux méthodes sont utilisables : la somme des températures et la somme des coefficients de $Q_{10}$. Dans une mise au point, DURAND (1967) remarque que la méthode des sommes de températures est surtout valable au printemps et en été; alors que les sommes des $Q_{10}$ ne conviennent pas quand les températures moyennes sont élevées. A partir de cette remarque et de l'étude des deux méthodes, nous avons finalement retenu la somme des températures. L'étude de la relation liant le coefficient $k$ à la température moyenne journalière nous a déjà permis de déterminer le seuil apparent de végétation des espèces étudiées.

(1). $\Delta 1$ représente l'accroissement de longueur pendant le temps $\Delta \mathrm{t}$. $\Delta \mathrm{t}$ est égal à 24 heures. 


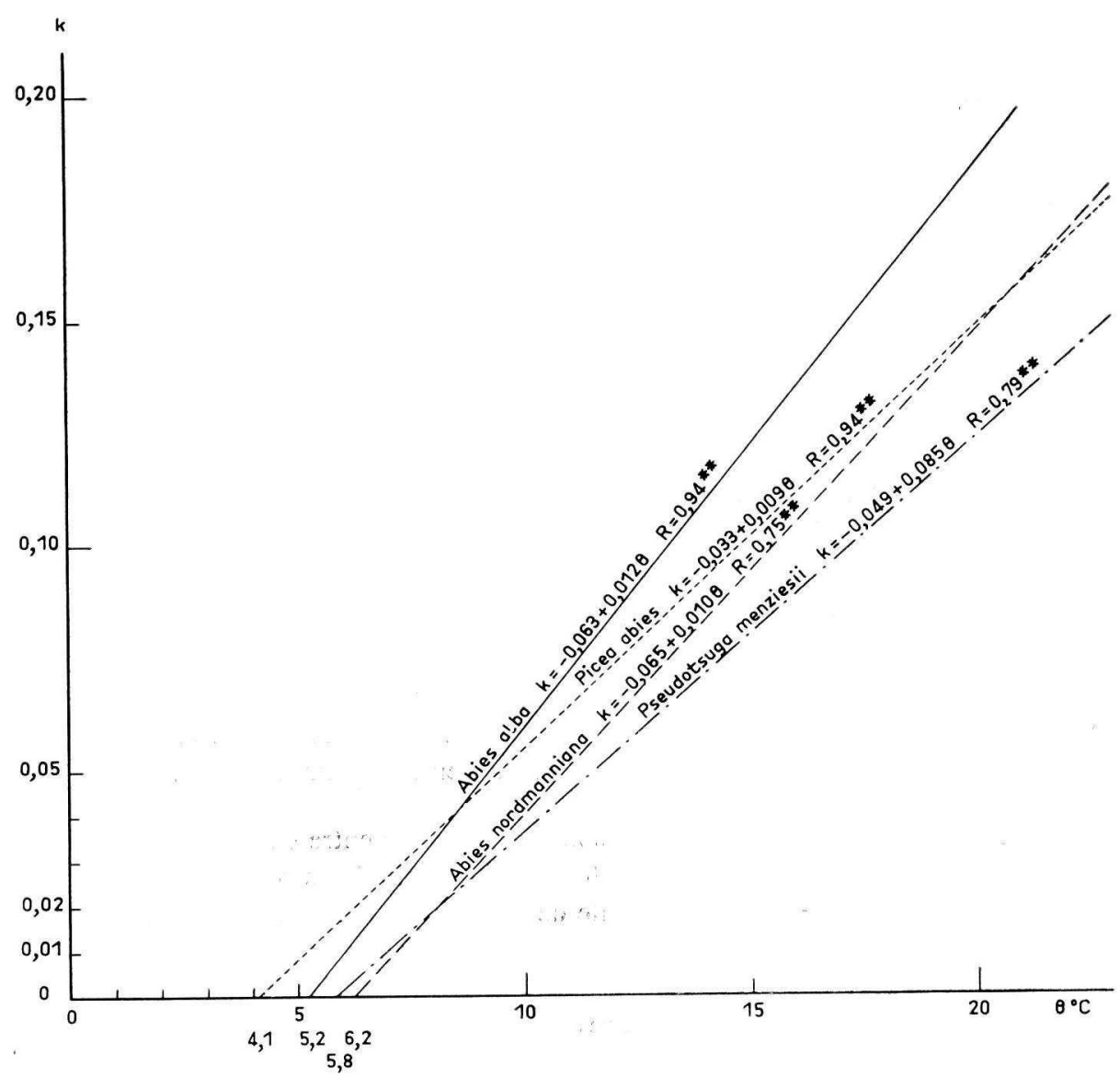

FIG. 3. - Variation du coefficient de croissance en fonction de la température moyenne journalière

Les sommations de températures ont été faites à partir des températures moyennes journalières et en utilisant l'expression suivante (Bidabe, 1967) :

$$
\begin{aligned}
&(\Theta \max -s)+(\Theta \min -s) \Theta \max : \text { température maximum } \\
& \Theta \min : \text { température minimum } \\
& s \quad \text { : seuil apparent de végétation }
\end{aligned}
$$

avec la condition suivante : $\Theta \max -s$ ou $\Theta \min -s=0$ quand $\Theta \max$ ou $\Theta \min \leqslant s$. La sommation a été effectuée pour chaque espèce à partir du stade $b 3{ }^{1}$ ) du débourrement, pour les différents milieux et années.

(1). Ce stade correspond à la phase de développement où les aiguilles sont partiellement libérées. Le stade réel de débourrement se situe entre le stade $b 2$ et le stade $b 3$, au moment de l'éclatement du Bourgeon (Aussenac, 1968). 
Les figures 4, 5, 6 et 7 montrent en exemple les courbes obtenues. En fait, il s'agit ici encore de courbes caractéristiques en « $S$ ». La partie inférieure de ces courbes n'est pas représentée sur ces figures car le début véritable de la croissance s'effectue au stade $b 2,5$, or, pour des raisons pratiques, nous sommes partis du stade $b 3$ ultérieur mais plus facile à préciser, car il représente un stade phénologique observable.

L'intégration de l'équation proposée par Robertson :

$$
\begin{gathered}
\frac{\Delta l}{\Delta t}=k l\left(1-\frac{l}{\mathrm{~L}}\right) \text { conduit aux équations (Durand, 1967) : } \\
\log \frac{l}{\mathrm{~L}-l}=k(t-t o) \\
l=\frac{\mathrm{L}}{1+e^{-k(t-t o)}} \text { avec to pour } l=\frac{\mathrm{L}}{2}
\end{gathered}
$$

Cette équation peut être retenue pour ajuster les courbes de croissance.

En fait, la variable temps doit être remplacée par la somme des températures. GesLiN (1944) a montré que cette transformation était valable. D'autres types de fonctions ont été proposés sans que pour autant l'ajustement soit meilleur. (RICKLEFFs, 1967).

Les courbes que nous avons obtenues sont comparables entre elles bien que, évidemment, elles correspondent à des croissances finales différentes. Elles présentent une portion qui peut être assimilée à une droite. La pente de cette droite ou plus précisément la pente de la tangente au point d'inflection peut être, avec une bonne approximation, considérée comme la même $\left({ }^{1}\right)$ pour toutes les courbes d'une même essence. Ce fait avait déjà été remarqué par GESLIN (1944). Les coefficients angulaires sont les suivants :

$$
\begin{array}{ll}
\text { - Abies alba } & 0,332 \\
\text { - Abies nordmanniana } & 0,308 \\
\text { - Picea abies } & 0,336 \\
\text { - Pseudotsuga menziesii } & 0,513
\end{array}
$$

On remarque que la valeur la plus élevée revient au Douglas, suivent ensuite l'Épicea et le Sapin pectiné, enfin le Sapin de Nordmann.

Ces résultats suggèrent que les potentialités de croissance différents de ces espèces, sont conditionnés par ces mécanismes d'action de la température.

L'accomplissement de tel ou tel cycle d'une phase de développement, en l'occurrence la croissance en hauteur, peut être relié aux sommes de température. D'après l'ensemble

(1). En réalité, il est probable que la pente n'est pas tout à fait constante et que la croissance ne suit pas tout à fait la loi logistique. 


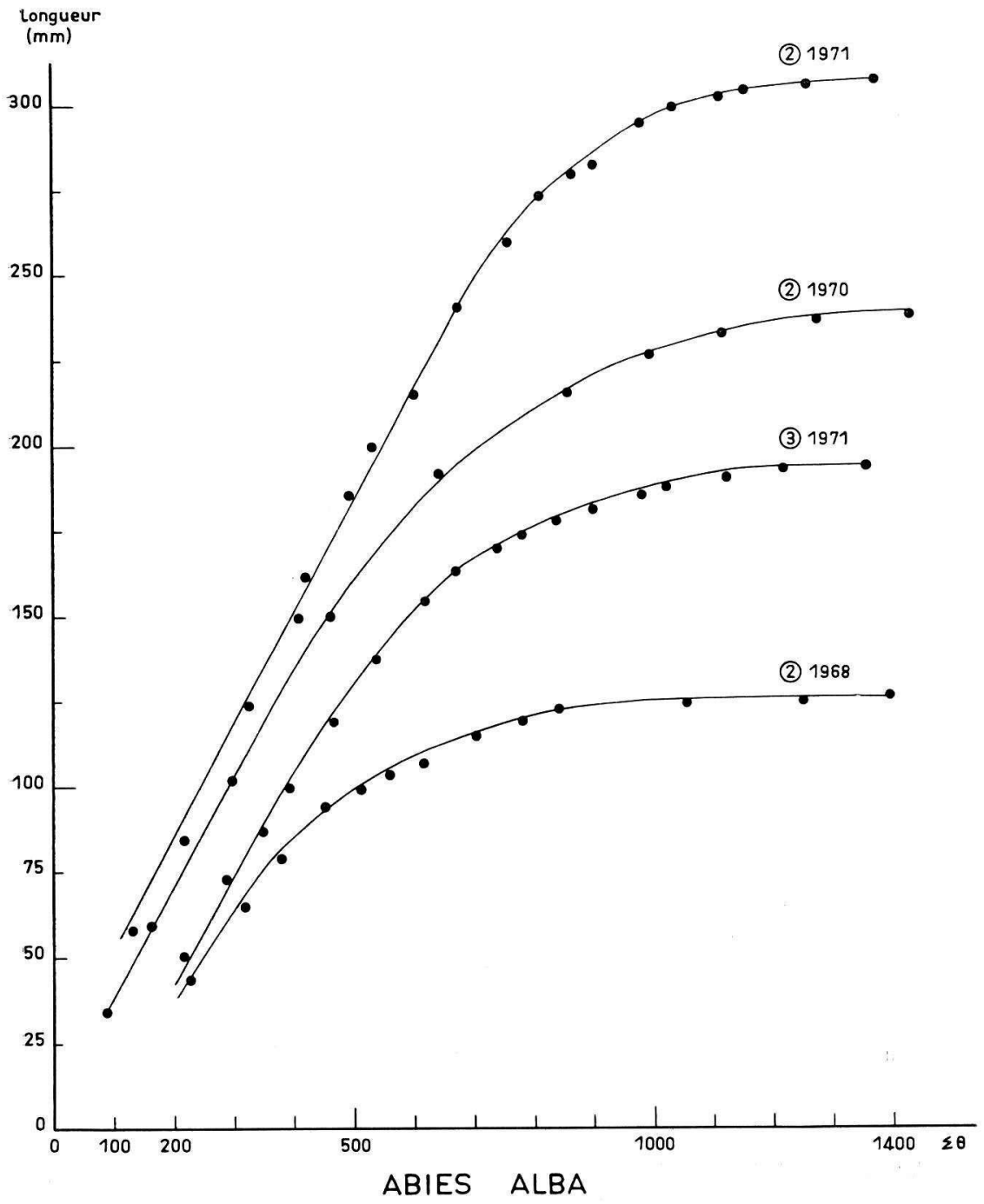

FIG. 4. - Variation de la longueur de la pousse en fonction des sommes de température: 2 : clairière, 3 : coupe rase 


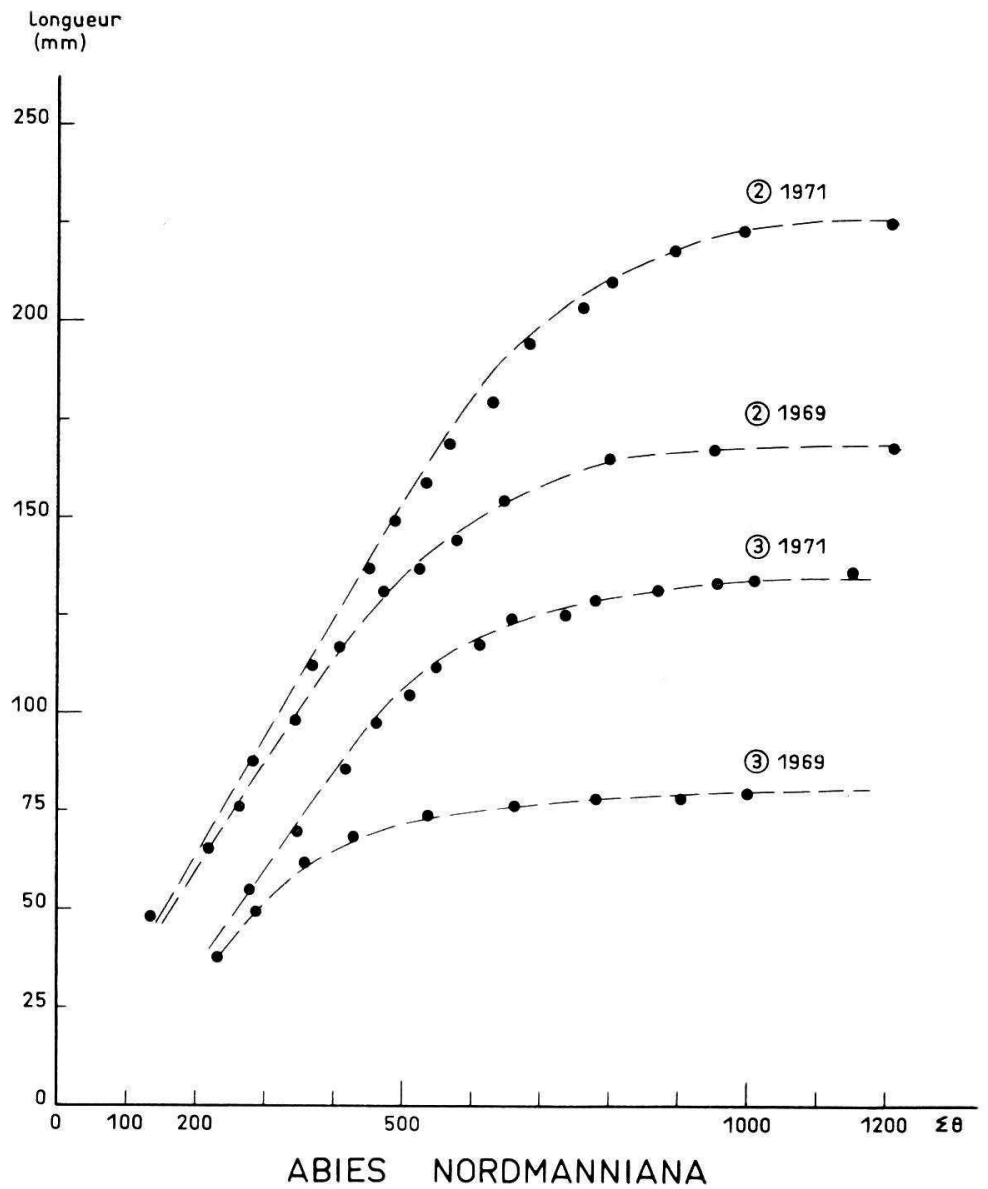

FIG. 5. - Variation de la longueur de la pousse en fonction des sommes de température: 2 : clairière, 3 : coupe rase 


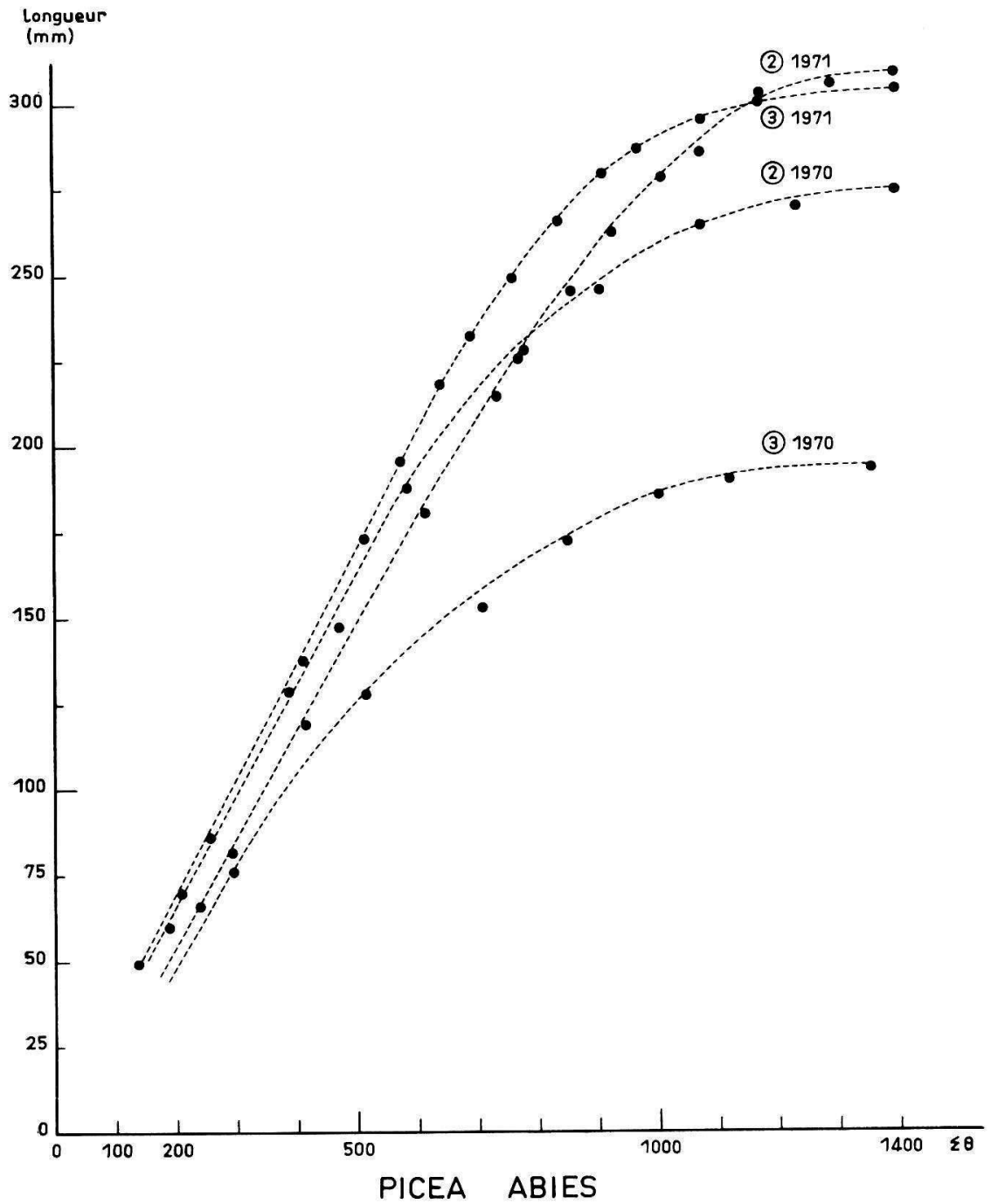

FIG. 6. - Variation de la longueur de la pousse en fonction des sommes de température : 2 : clairière, 3 : coupe rase 


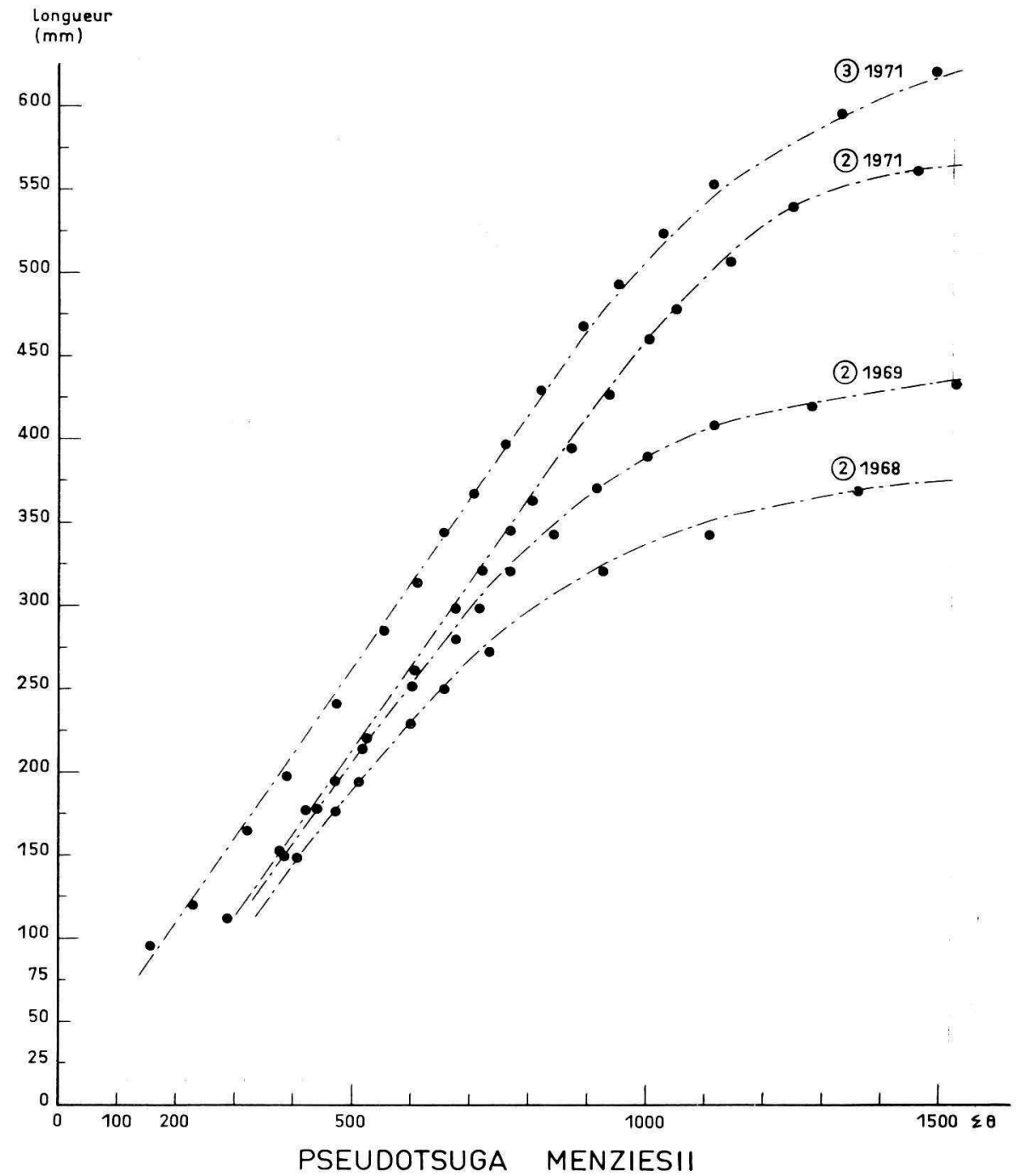

Fig. 7. - Variation de la longueur de la pousse en fonction des sommes de température: 2 : clairière, 3 : coupe rase 
des mesures effectuées, les sommes de température nécessaires à la réalisation de la croissance totale peuvent être estimées ( $\left.{ }^{1}\right)$ à :

- 1240 pour Abies alba (seuil de sommation 5,2 ${ }^{\circ} \mathrm{C}$ )

- 1200 pour Abies nordmanniana (seuil de sommation $6,2{ }^{\circ} \mathrm{C}$ )

- 1400 pour Picea abies (seuil de sommation $4,1{ }^{\circ} \mathrm{C}$ )

- 1600 pour Pseudotsuga menziesii (seuil de sommation $5,8^{\circ} \mathrm{C}$ )

Dans le détail des milieux et des années, il est très difficile d'estimer la diversité des sommes de température nécessaires. En effet, compte tenu de la précision des mesures et de la difficulté d'estimer la croissance en fin d'accroissement, il n'est pas facile d'estimer avec précision la date de l'arrêt de l'allongement de la flèche. Or, à ce moment-là de la saison de végétation, les températures sont généralement élevées pour des accroissements faibles, donc les erreurs sur les sommes peuvent être relativement importantes.

\section{4. - DISCUSSION ET CONCLUSION}

L'étude de la croissance en hauteur en fonction de la température nous a permis de régulariser les courbes et de les comparer entre elles. Les résultats obtenus pour les seuils apparents de végétation font apparaître des différences intéressantes (bien que pas toutes significatives) entre les espèces étudiées. Les valeurs trouvées sont cohérentes avec ce que l'on connaît de la biologie de ces essences. L'Épicéa apparaît comme la « plus septentrionale » d'entre elles. Au Canada, Worrall (1973) avance pour cette espèce un seuil apparent de $2,6{ }^{\circ} \mathrm{C}$. Cette valeur, plus faible que celle que nous avons trouvée, pourrait peut-être s'expliquer par une provenance différente des arbres ou par une répartition différente des températures.

Abies nordmanniana quant à lui se différencie nettement du Sapin pectiné par un seuil de végétation plus élevé.

Les courbes de croissance en fonction des sommes de température présentent une caractéristique commune : la pente de la tangente au point d'inflexion pour une essence donnée, peut être considérée avec une bonne approximation comme constante, sensiblement à une condition toutefois, c'est que la croissance ne soit pas extrêmement réduite, comme c'est le cas pour la coupe d'abri. L'influence relative des autres facteurs écologiques (lumière et eau) est alors écrasante et masque le rôle de la température.

Il est alors possible d'imaginer que cette caractéristique des courbes de croissance est fonction du génotype. Plus cette constante est élevée et plus les potentialités de croissance en hauteur sont grandes. Les résultats obtenus montrent que le Douglas a la potentialité la plus grande, suivi de l'Épicea et du Sapin pectiné, le Sapin de Nordmann aurait la potentialité la plus faible des quatre essences considérées ici.

Bien entendu, au sein de chaque espèce, il serait intéressant de savoir si cette constante varie avec la provenance des arbres. Autre question : cette caractéristique varie-t-elle dans

(1). A compter du stade $b 3$ du débourrement et d'après 5 ans d'observations. On pourra bien entendu diviser ces valeurs par deux pour les comparer avec les chiffres obtenus par les auteurs qui ont utilisé les températures moyennes pour effectuer les sommations. 
le temps avec l'âge? : cela semble peut probable. Ce qui varie, et nous l'avons constaté, c'est seulement l'ordonnée à l'origine de la tangente au point d'inflection. L'explication est simple : plus le végétal se trouvera dans de meilleures conditions de croissance, plus la courbe de l'allongement en fonction des sommes de température se rapprochera de la courbe « idéale » de croissance maximum, obtenue quand tous les autres facteurs écologiques sont à l'optimum pour l'espèce considérée.

On voit alors l'intérêt de déterminer expérimentalement la loi physiologique de la croissance en hauteur en fonction des sommes de température, tous les autres facteurs écologiques étant à l'optimum pour une espèce déterminée.

Reçu pour publication en décembre 1974.

\section{SUMMARY}

STUDY OF THE HEIGHT GROWTH IN SOME CONIFERS INFLUENCE OF TEMPERATURE

Height growth of four conifers (Abies alba, Abies nordmanniana, Picea abies, Pseudotsuga menziesii) growing in different natural environmental conditions has been studied and measured twice a week.

The relationship between the mean air temperature and the growth coefficient $k$ was studied

$$
k=\frac{1}{l\left(1-\frac{l}{\mathrm{~L}}\right)} \cdot \frac{\Delta l}{\Delta t}
$$

$l$ beeing the lenght ot the shoot at a given moment $t$ and

$\mathrm{L}$ the maximum shoot lenght.

This coefficient is equal to nought for the following temperatures :

$\begin{array}{ll}\text { Abies alba } & 5,2{ }^{\circ} \mathrm{C} \\ \text { Abies nordmanniana } & 6,2{ }^{\circ} \mathrm{C} \\ \text { Picea abies } & 4,1{ }^{\circ} \mathrm{C} \\ \text { Pseudotsuga menziesii } & 5,8^{\circ} \mathrm{C}\end{array}$

These are the minimum temperature (threshold temperature) required for shoot growth

This minimum is highest for Abies nordmanniana which is the most " southern " species which has been studied and the lowest for Picea abies which is most « northern » species.

The relationships between shoot growth and the sum of temperatures above threshold temperature has been studied for each species. We got classical $« S »$ shaped curves. The slope $(\alpha)$ of the tangent at the inflexion point is almost constant for a given species.

$\begin{array}{cc}\text { for } \text { Abies alba } & 0,332 \\ \text { Abies nordmanniana } & 0,308 \\ \text { Picea abies } & 0,336 \\ \text { Pseudotsuga menziesii } & 0,513\end{array}$

The results indicate that the growth potentialities of the different species are depending both of the threshold temperatures and $\alpha$. Douglas fir has the best potentiality, followed by Picea abies, Abies alba and Abies nordmanniana. 


\section{ZUSAMMENFASSUNG}

\section{HOHENWACHSTUM UND LUFTTEMPERATUR}

Der Autor untersuchte das Höhenwachstum von vier verschiedenen Nadelholzarten (Abies alba, Abies nordmanniana, Picea abies, Pseudotsuga menziesii) unter mikroklimatsch unterschiedlichen Bedingungen.

Der Höhenzuwachs wurde zweimal wöchentlich gemessen und eine Beziehung zwischen der mittleren Lufttemperatur und einem Zuwachskoeffizienten berechnet.

$$
k=\frac{1}{l\left(1-\frac{l}{\mathrm{~L}}\right)} \cdot \frac{\Delta l}{\Delta t}
$$

$l$ : Sprosslänge zum Zeitpunkt $t$

L : Sprosslänge am Ende der Wachstumsperiode.

Dieser Zuwachskoeffizient wird bei folgenden Temperaturen annulliert :

$\begin{array}{ll}\text { Abies alba } & 5,2^{\circ} \mathrm{C} \\ \text { Abies nordmanniana } & 6,2^{\circ} \mathrm{C} \\ \text { Picea abies } & 4,1^{\circ} \mathrm{C} \\ \text { Pseudotsuga menziesii } & 5,8^{\circ} \mathrm{C}\end{array}$

Diese Temperaturwerte können als die augenscheinliche Vegetationsschwelle bezeichnet, werden. Abies nordmanniana als die "südlichste » Holzart hat die höchste Vegetationsschwelle, während Picea abies, als nördlichste Art, die niedrigste Vegetationsschwelle aufweist.

Das Höhenwachstum wurde im weiteren als Funktion der Temperatursummen dargestellt, welche ausgehend von den Holzartentypischen Vegetationsschwellwerten berechnet wurden. Der so erhaltene Kurvenverlauf weist die klassische « $\mathrm{S}$ » Form auf. Die Steigung der Wendetangente kann als eine holzartenspezifische Konstante aufgefasst werden. Es ergeben sich die folgenden Konstanten :

$\begin{array}{ll}\text { Abies alba } & 0,332 \\ \text { Abies nordmanniana } & 0,308 \\ \text { Picea abies } & 0,336 \\ \text { Pseudotsuga menziesii } & 0,513\end{array}$

Diese Ergebnisse lassen vermuten, dass das potentielle Höhenwachstum dieser vier verschiedenen Holzarten durch die obigen Temperatureinwirkungen bedingt wird. Die Douglasie hat das höchste Wuchspotential, während die Nordmann Tanne an letzter Stelle steht.

\section{RÉFÉRENCES BIBLIOGRAPHIQUES}

Aussenac, G., 1968. - Observations sur les effets d'une gelée tardive. Revue Forestière Française, 3, 204241.

Aussenac G., 1973. - Climat, microclimat et production ligneuse. Ann. Sci. Forest. 30 (3) 239-258.

BidaBe B., 1967. - Action de la température sur l'évolution des bourgeons de pommier et comparaison des méthodes de contrôle de l'époque de floraison. Ann. Physiol. Vég., 9 (1), 65-86.

Decourt N, 1971. - Comparaison des équations de régression. Application au cubage des peuplements d’Épicéa commun. Ann. Sci. Forest., 28 (1) 51-58.

Durand R., 1967. - Action de la température et du rayonnement sur la croissance. Ann. Physiol. vég.,

9 (1) 5-27. 
Durand R., de Parcevaux S., Roche P., 1967. - Action de la température sur la croissance et le développement du lin. Ann. Physiol. Vég. 9 (1) 87-105.

GesLin M., 1944. - Étude des lois de croissance d'une plante en fonction des facteurs du climat. Thèse Faculté des Sciences, Paris, 115 p.

RickLefs R. E., 1967. - A graphical method of fitting equations to growth curves. Ecology, 48 (6), 978-983.

Worrall J., 1973. - Seasonal, daily and hourly growth of height and radius in Norway Spruce. Canadian Journal of Forest Research 3, 501-511. 